\title{
Resolved Photons and BFKL-type Signatures in Deep Inelastic Scattering ${ }^{a}$
}

\author{
H. Jung \\ University of Lund, Department of Physics, Sweden
}

\begin{abstract}
The concept of resolved virtual photons in addition to direct deep inelastic $e p$ scattering is used to simulate the $2+1$ jet - rate and the forward jet cross section, which cannot be described by direct LO/NLO processes. With standard DGLAP evolution of the parton densities of the virtual photon and the proton the HERA data can be described, provided a sufficiently large scale for the hard scattering matrix element is used.
\end{abstract}

\section{Introduction}

At HERA the measurement of the inclusive forward jet production 1 目 in the proton direction in deep inelastic events at low $x$ and large $Q^{2}$ is believed to be one of the mpst sensitive measurements for BFKL dynamics. The measured cross section 3 cannot be explained by standard deep inelastic scattering models

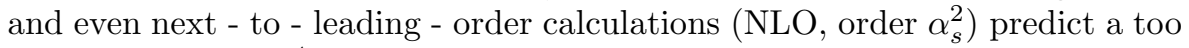
small cross sectiont.

The measured 0 fractional $2+1$ jet rate $R_{2+1}=\frac{\sigma^{2+1}}{\sigma^{t o t}}$, where two high $p_{T}$ jets come from the hard QCD scattering and the " +1 " stands for the remnant jet, seems to be a factor of $\sim 2$ larger than predictions from standard deep inelastic scattering (DIS) models.

However both measurements are rather well described by the Color - Dipole - Model (CDM) in its implementation in ARIADNE

In this article we study to what extent the contribution of virtual resolved photons in addition to the standard DIS matrix element calculations (labeled as direct contributions) can reproduce the data. The studies are performed with the RAPGAPU Monte Carlo event generator which for the direct processes agrees rather well with the LEPTOG Monte Carlo program.

\section{Resolved Photons in DIS}

Resolved photon processes play an important role in photo-production, when high $p_{T}$ jets are observed. Any internal structure of the proton as well as of the photon can be resolved as long as the scale $\mu^{2}$ of the hard subprocess, which is

\footnotetext{
${ }^{a}$ To be published in Proceedings of the Madrid low $x$ Workshop, Miraflores de la Sierra, 1997
} 
of the order of $p_{T}^{2}$, is larger than the inverse radius of the proton $1 / R_{p}^{2} \sim \Lambda_{Q C D}^{2}$ and the photon $1 / R_{\gamma}^{2} \sim Q^{2}$.

Resolved photon processes in DIS are calculated here using the Equivalent Phpton Approximation, giving the flux of virtual transverse polarized photons 0 together with the on shell matrix elements for the partonic subprocesses. The structure of the virtual photon is given by parametrisations of the parton densities 10 .11. $12 x_{\gamma} f_{\gamma}\left(x_{\gamma}, \mu^{2}, Q^{2}\right)$, now depending on the scale $\mu^{2}$ and the photon virtuality $Q^{2}$. Direct deep inelastic scattering and resolved virtual photon processes are simulated using the RAPGAP Monte Carlo event generator . The hard subprocesses for resolved photons implemented in RAPGAP are: $g g \rightarrow q \bar{q}, g g \rightarrow g g, q g \rightarrow q g, q \bar{q} \rightarrow g g, q \bar{q} \rightarrow q \bar{q}$ and $q q \rightarrow q q$.

In DIS, resolved photon processes 13.10 can play a role when the scale $\mu^{2}$ of the hard subprocess is larger than the virtuality of the photon $Q^{2}: \mu^{2}>Q^{2}$. A typical NLO diagram like $\gamma^{*} g \rightarrow q \bar{q} g$ occurs also in the resolved photon picture, via the subprocess $\bar{q} g \rightarrow \bar{q} g$ with the photon splitting $\gamma^{*} \rightarrow q \bar{q}$. When including resolved photon processes in a NLO calculation special care has to be taken to avoid double counting 4 . In leading order there is no double counting when adding resolved photons to direct processes since they have different final states (three parton and two parton).

\subsection{Scales, $\alpha_{s}$ and Parton Distribution Functions}

In leading order $\alpha_{s}$ processes the renormalisation scale $\mu_{R}$ and factorization scale $\mu_{F}$ are not well defined, and any choice of $\mu_{R}^{2}=\mu_{F}^{2}=Q^{2}, p_{T}^{2}, Q^{2}+$ $p_{T}^{2}$, and multiples thereof, is reasonable. There are essentially two competing effects: a larger scale gives smaller $\alpha_{s}\left(\mu^{2}\right)$, but a larger parton density $x f\left(x, \mu^{2}\right)$ at fixed $x$. The net effect depends on the details of the interaction.

The effect of different choices of the scale $\mu^{2}=Q^{2}+4 \cdot p_{T}^{2}, \mu^{2}=Q^{2}+p_{T}^{2}$, $\mu^{2}=4 \cdot p_{T}^{2}$, or $\mu^{2}=p_{T}^{2}$ is discussed in the following.

We use the GRV 94 HO (DIS) parametrisation of the proton parton densities 1516 together with the SaSgam 2D 11 parametrisation for the partons in the virtual photon. Other choices like the GRV LO parton density of the real photon together with the virtual photon suppression factor of Drees - Godbole 12 give similar results.

\section{Forward Jets}

We study the HERA data on high $p_{T}$ "forward" jet production at low $x$ and large $Q^{2}$ in the context of resplved virtual photons. The cuts are taken from the preliminary $\mathrm{H} 1$ analysis $\mathrm{B}^{\mathrm{B}} \mathrm{y}>0.1, E_{e}^{\prime}>11 \mathrm{GeV}, 160^{\circ}<\theta_{e}<173^{\circ}$, 

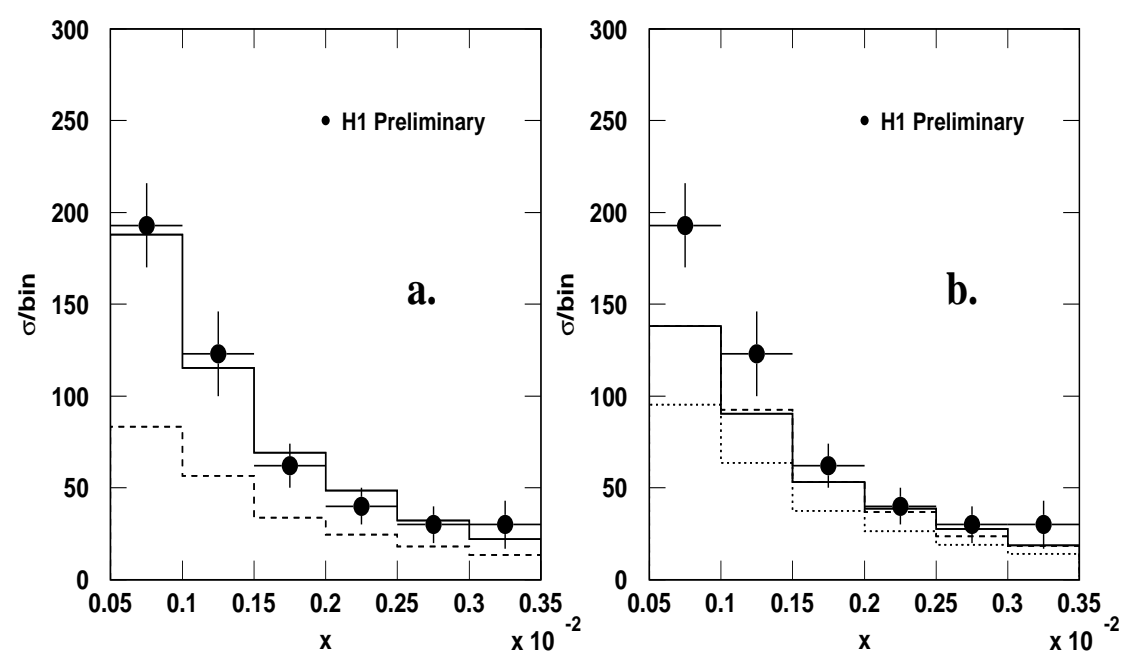

Figure 1: The forward jet cross section as a function of $x$ using the cots of the H1 collaboration specified in the text. The data points are preliminary $\mathrm{H} 1$ data 3 . In $1 a_{\text {. }}$ the dashed line is the RAPGAP prediction for direct DIS processes and the solid line is the RAPGAP prediction for the sum of direct and resolved contributions (using $\mu^{2}=Q^{2}+4 p_{T}^{2}$, GRV $\mathrm{HO}$ for the proton and SaSgam 2 DIS for the virtual photon). In 1b.) the solid line is the RAPGAP prediction for the sum of direct and resolved contributions with $\mu^{2}=Q^{2}+p_{T}^{2}$, the dashed line with $\mu^{2}=4 p_{T}^{2}$ and the dotted line with $\mu^{2}=p_{T}^{2}$. The difference between solid and dashed lines is hardly visible.

$7^{\circ}<\theta_{\text {jet }}<20^{\circ}, E_{\text {jet }}>28.7 \mathrm{GeV}$, and $p_{T}^{\text {jet }}>3.5 \mathrm{GeV}$ in the laboratory frame. The observed forward jet is required to have sufficiently large $E_{j e t}$ and $p_{T}^{\text {jet }}$ in order to rely on well measured parton distributions in the proton. A $p_{T}$ of the jet is required to be of the same order as $Q^{2}\left(0.5<p_{T}^{2} / Q^{2}<2\right)$ to suppress the contribution from direct LO processes which are governed by DGLAP evolution. The event topology is a small $x$ deep inelastic event with an energetic high $p_{T}$ jet in the forward (proton) direction. In Fig. 1 $a$.) preliminary data of the H1 collaboration 3 are shown together with the RAPGAPG Monte Carlo prediction for standard DIS processes (direct) and the sum of direct and resolved processes, using the scale $\mu^{2}=Q^{2}+4 p_{T}^{2}$. We note that the dominant contribution of resolved photon processes to the forward jet cross section comes from $q g \rightarrow q g$.

With a scale $\mu^{2}=Q^{2}+4 p_{T}^{2}$ (with $p_{T}$ defined in the parton parton cms) a perfect description of the data (Fig. 1 1 a. $)$ is achieved. The effect of different choices of the scale $\mu^{2}$ is shown in Fig. 1 $1 \beta$.). We observe no difference using 
$\mu^{2}=Q^{2}+p_{T}^{2}$ or $\mu^{2}=4 p_{T}^{2}$. In general a less hard scale results in a smaller cross section and worse agreement with the data at small $x$ values. Using $\mu^{2}=p_{T}^{2}$ the predicted cross section falls well below the measurement. This is because the condition $\mu^{2}=p_{T}^{2}>Q^{2}$ together with $0.5<Q^{2} / p_{T}^{2}<2$ leaves only little space for resolved photon contributions. However with the concept of resolved virtual photons we obtain a improved description of the data for all choices of the scale $\mu^{2}$.

\section{$42+1$ jet rates in DIS}

Dijets $(2+1-$ jets $)$ are searched for with a cone jet algorithm by requiring a $p_{T}^{\text {jets }}>5 \mathrm{GeV}$ in the hadronic center of mass frame, $Q^{2}>5 \mathrm{GeV}^{2}, y>0.05$, $E^{\prime}>11 \mathrm{GeV}$ and $156^{\circ}<\theta_{e}<173^{\circ}$ according to the preliminary $\mathrm{H} 1$ - analysis I. $^{2}$ Since in that analysis $p_{T}^{2}>Q^{2}$ in the region $Q^{2}<25 \mathrm{GeV}^{2}$, resolved photon interactions can be expected to play a significant role. It has been observed 6 , that direct LO Monte Carlos like RAPGAP direct and LEPTO as well as NLO calculations are unable to describe the data especially in the low $Q^{2}$ region of $Q^{2}<30 \mathrm{GeV}^{2}$. However ARIADNE agrees with the data remarkably well. In Fig. 2a.) and $b$.) the H1 measurement ${ }^{2}$ of $R_{2+1}$ as a function of $Q^{2}$ and $x$ is shown together with the direct contribution and the sum of direct and resolved photons obtained with RAPGAP. The parameter setting is the same as for the forward jet analysis. We find remarkable agreement (using $\mu^{2}=Q^{2}+4 p_{T}^{2}$ ) between the data and the Monte Carlo prediction showing that especially in the region of phase space where $Q^{2}<p_{T}^{2}$, resolved photons play an important

role. Note that here also the dominant contribution from resolved photon processes comes from $q g \rightarrow q g$.

We have checked $R_{2+1}$ as a function of $Q^{2}$ and $x$ also for different scales $\mu^{2}=Q^{2}+p_{T}^{2}, \mu^{2}=4 p_{T}^{2}$ and $\mu^{2}=p_{T}^{2}$ used for the resolved photon contribution. The contribution of resolved photons to $R_{2+1}$ is larger for the largest scale $\mu^{2}=Q^{2}+4 p_{T}^{2}$, since there is a larger phase space for DGLAP evolution in the photon. The results are shown in Fig. 2 2 c.) and $d$.) Again we observe the same trend, that including resolved photons in DIS, the description of the measurements is considerably improved for all different choices of the scales $\mu^{2}$.

\section{Discussion}

A scale dependence is expected in all leading order calculations, but it becomes more important when the concept of resolved photons is applied to DIS: in order to resolve any internal structure of the virtual photon, the scale involved 

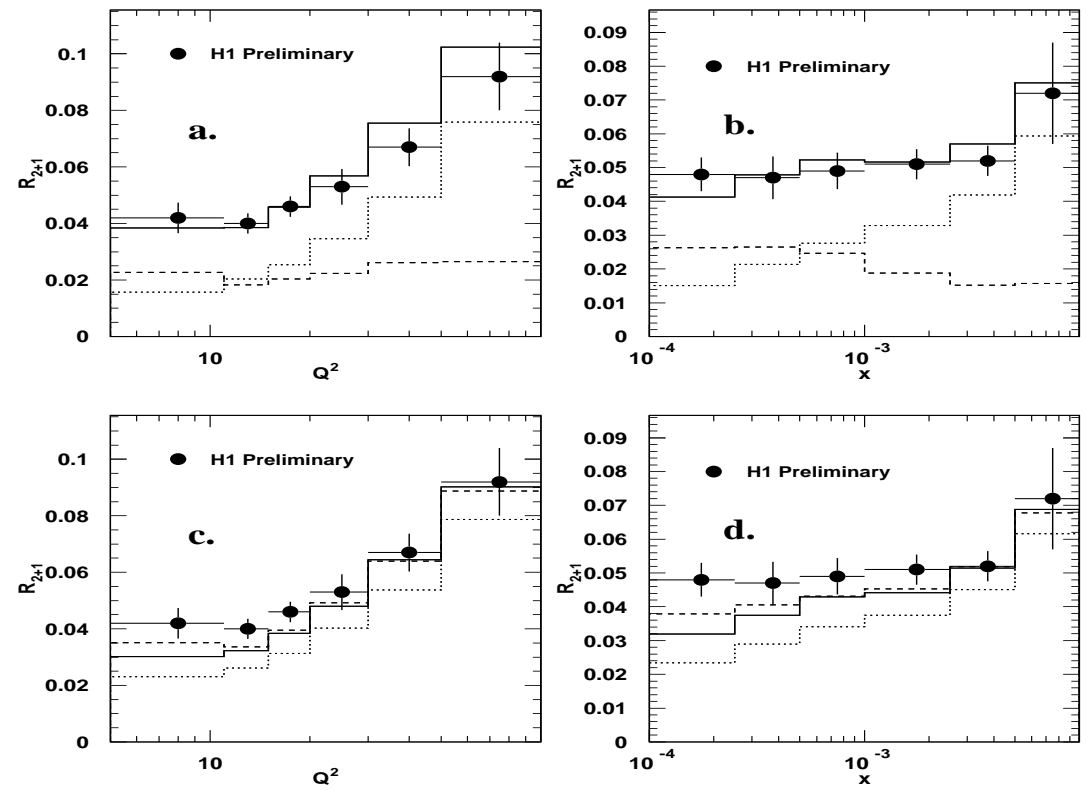

Figure 2 The $2+1$ jet ratio $R_{2+1}$ as a function of $Q^{2}$ and $x$. The data points are preliminary $\mathrm{H} 1$ data5. In $2 a$.) and $b$.) the dotted line is the RAPGAP prediction for the direct contribution, the dashed line for resolved photon contribution and the solid line is the prediction for the sum of direct and resolved contributions (using $\mu^{2}=Q^{2}+4 p_{T}^{2}$, GRV HO for the proton and SaSgam 2 DIS for the virtual photon). In $2 c$.) and $d$.) the solid line is the RAPGAP prediction for the sum of direct and resolved contributions with $\mu^{2}=Q^{2}+p_{T}^{2}$, the dashed line with $\mu^{2}=4 p_{T}^{2}$ and the dotted line with $\mu^{2}=p_{T}^{2}$.

has to be larger than the virtuality of the photon $Q^{2}: \mu^{2}>Q^{2}$. Using a large scale like $\mu^{2}=Q^{2}+4 p_{T}^{2}$ results in a larger magnitude of the parton density, both on the photon and proton side, at fixed values of fractional parton momenta $\xi_{i}$, due to the larger phase space for DGLAP evolution. This effect is compensated to some extent by the smaller value of $\alpha_{s}$ at the same scale.

In case of the forward jet cross section the stronger scale dependence becomes understandable because of the requirement $0.5<Q^{2} / p_{T}^{2}<2$. Although here $p_{T}$ is measured in the lab system this cut restricts severely the phase space for resolved photons.

With the concept of resolved photons, experimentally observed phenomena in DIS, such as the forward jet cross section and the $2+1$ jet rate can be well described, provided a reasonable large scale for the hard scattering process 
is used. This concept and its implementation into a full hadron level Monte Carlo generator can be seen as a model alternative to BFKL calculations 17. The next step would be to search for regions in phase space where the two descriptions do not agree but the data can only be described by one of them.

\section{Acknowledgment}

I am grateful for many fruitful discussions with L. Jönsson and H. Küster.

Without the great time and fun I still have with Antje it would not have been possible to search for free living pomerinos on the Canary Islands.

\section{References}

1. A. Mueller, Nucl. Phys. B (Proc. Suppl) 18C (1990) 125.

2. A. Mueller, J. Phys. G 17 (1991) 1443.

3. H1 Collaboration, C. Adloff et al., Contributed paper, pa 03-49, ICHEP96, Warsaw, Poland, July 1996 (unpublished).

4. E. Mirkes, D. Zeppenfeld, Fixed-Order QCD Background to BFKL Dynamics in Forward Jet Production, in Proceedings of the Workshop on Future Physics at HERA, edited by A. De Roeck, G. Ingelman, R. Klanner (1995).

5. H1 Collaboration, T. Ahmed et al., Contributed paper, Abstract 247, HEP97, Jerusalem, Israel, August 1997 (unpublished).

6. L. Lönnblad, Comp. Phys. Comm. 71 (1992) 15, ARIADNE 4.08.

7. H. Jung, The RAPGAP Monte Carlo for Deep Inelastic Scattering, version 2.06, http://www-h1. desy.de/ jung/rapgap.html.

8. G. Ingelman, A. Edin, J. Rathsman, Comp. Phys. Comm. 101 (1997) 108, LEPTO 6.5.

9. H. Jung, Comp. Phys. Comm. 86 (1995) 147.

10. M. Glück, E. Reya, M. Stratman, Phys. Rev. D 54 (1996) 5515.

11. G. Schuler, T. Sjöstrand, Phys. Lett. B 380 (1996) 105.

12. M. Drees, R. Godbole, Phys. Rev. D 50 (1994) 3124.

13. J. Chyla, J. Cvach, Virtual Photon Structure from jet production at HERA, in Proceedings of the Workshop on Future Physics at HERA, edited by G. Ingelman, A. De Roeck, R. Klanner (Hamburg, 1996).

14. M. Klasen, G. Kramer, B. Potter, hep-ph/9703302 .

15. M. Glück, E. Reya, A. Vogt, Z. Phys. C 53 (1992) 127.

16. M. Glück, E. Reya, A. Vogt, Phys. Lett. B 306 (1993) 391.

17. J. Bartels et al., Phys. Lett. B384 (1996) 300, hep-ph/9604272. 